\title{
DFG setzt neue Akzente für Open Access
}

\author{
Förderung von Publikationskosten und gezielte Unterstützung \\ für wissenschaftsfreundliche Publikationsinfrastrukturen / \\ Themen- und Hintergrundinformationen auf der DFG-Webseite
}

https://doi.org/10.1515/bd-2021-0032

Die Deutsche Forschungsgemeinschaft (DFG) verstärkt ihre Bemühungen um den freien Zugang zu Publikationen und anderen Forschungsergebnissen im Netz. Um Open Access zu unterstützen und diesen bedarfsgerecht an die sich ändernden Anforderungen von Wissenschaft und Forschung anzupassen, hat die DFG weitere Maßnahmen beschlossen und umgesetzt. Diese sind miteinander vernetzt und reichen von verbesserten Rahmenbedingungen über die Finanzierung von Publikationskosten und den Aufbau einer wissenschaftsadäquaten Publikationsinfrastruktur bis zur Mitwirkung in nationalen und internationalen Arbeitsgruppen.

„Ich freue mich sehr, dass die DFG ihre Unterstützung des Open Access mit diesen Maßnahmen konsequent ausbaut“, unterstreicht DFG-Präsidentin Professorin Dr. Katja Becker. „Die Coronavirus-Pandemie hat einmal mehr gezeigt, wie wichtig der offene und rasche Austausch von wissenschaftlichen Ergebnissen ist, um sowohl Erkenntnisfortschritt als auch gesellschaftliche Entwicklungen voranzubringen. Die DFG möchte Wissenschaftlerinnen und Wissenschaftler aller Disziplinen dabei bestmöglich unterstützen.“

Die DFG hat 2020 ihre Open Access-Policy nachjustiert. Wissenschaftlerinnen und Wissenschaftler werden nun aufgefordert, Ergebnisse aus DFG-geförderten Forschungsprojekten im Open Access zu publizieren. Um dieses Ziel zu erreichen, unterstützt die DFG mit ihren Förderprogrammen sowohl die Finanzierung von Publikationsgebühren als auch den Aufbau von geeigneten Publikationsinfrastrukturen.

Mit ihrem im Herbst 2020 eingeführten Programm „Open Access-Publikationskosten“ gewährt die DFG Zuschüsse zu den Gebühren für Veröffentlichungen. Dabei können sowohl die Gebühren für Zeitschriftenartikel als auch für Open Access-Monografien gefördert werden. Viele Universitäten und außeruniversitäre Forschungseinrichtungen sehen sich der finanziellen Herausforderung gegenüber, dass Verlage für die Veröffentlichung von Forschungsergebnissen im Open Access Gebühren verlangen. Das neue Programm soll die Einrichtungen und deren Wissenschaftlerinnen und Wissenschaftler bei der Open Access-Transformation unterstützen. 
Über die Finanzierung von Publikationsgebühren hinaus sind die verschiedenen fachwissenschaftlichen Communities in Deutschland auf die Weiterentwicklung wissenschaftsfreundlicher Standards und Infrastrukturen angewiesen. Mit dem neu akzentuierten Förderprogramm „Infrastrukturen für wissenschaftliches Publizieren" unterstützt die DFG die Open Access-Transformation durch den Aufund Ausbau geeigneter Publikationsinfrastrukturen und fördert damit auch die (Weiter-)Entwicklung struktureller Rahmenbedingungen des Publikationswesens.

Darüber hinaus engagiert sich die DFG in nationalen und internationalen Kontexten wie der Schwerpunktinitiative „Digitale Information“, Science Europe oder der Initiative Knowledge Exchange zum Thema Open Access. Die DFG ist zudem Ende 2020 der ENABLE!-Community beigetreten, die ein partnerschaftliches Netzwerk aufbaut, um Open Access-Publikationen in den Geistes- und Sozialwissenschaften auch durch die Entwicklung innovativer Co-Publishing-Modelle zu ermöglichen.

Alle genannten Maßnahmen werden auf einer neuen Webseite zum Thema „Open Access“ ausführlicher beschrieben. Die Seite bietet Hintergrundinformationen, skizziert wissenschaftspolitische Positionen der DFG und stellt die Förderangebote zur Unterstützung der Open Access-Transformation ebenso dar wie das Engagement der DFG in nationalen und internationalen Arbeitsgruppen.

Die DFG unterstützt Open Access bereits seit 2003. Open Access ermöglicht es Forscherinnen und Forschern, ihre eigenen Arbeiten weltweit zu verbreiten sowie die Publikationen anderer schnell und unmittelbar zu rezipieren, und zwar ohne dass technische, finanzielle oder organisatorische Barrieren die weitere Verbreitung oder Verwendung dieser Veröffentlichungen einschränken.

\section{Weiterführende Informationen:}

- Webseite Open Access der DFG: www.dfg.de/lis/openaccess

- Programm „Open Access-Publikationskosten“: www.dfg.de/foerderung/ programme/infrastruktur/lis/lis_foerderangebote/open_access_publika tionskosten

- Programm „Infrastrukturen für wissenschaftliches Publizieren“: www.dfg.de/ foerderung/programme/infrastruktur/lis/lis_foerderangebote/infrastruk turen_publizieren

- Webseite der ENABLE!-Community: https://enable-oa.org

\section{Fachliche Ansprechpersonen in der DFG-Geschäftsstelle:}

- Für das Programm „Open Access-Publikationskosten“: Dr. Angela Holzer, Wissenschaftliche Literaturversorgungs- und Informationssysteme

Tel.: +49 228 885-2568 | E-Mail: angela.holzer@dfg.de 
- Für das Programm „Infrastrukturen für wissenschaftliches Publizieren“: Dr. Juliane Kant, Wissenschaftliche Literaturversorgungs- und Informationssysteme

Tel.: +49 228 885-2009 | E-Mail: juliane.kant@dfg.de

\section{Medienkontakt:}

Dr. Rembert Unterstell, Presse- und Öffentlichkeitsarbeit der DFG

Tel.: +49 228 885-2109 | E-Mail: presse@dfg.de 